\title{
Determining the complexity of multi-component conformal systems: A platoon-based approach
}

\author{
Caglar Kosun $^{\mathrm{a}, *}$, Serhan Ozdemir ${ }^{\mathrm{b}}$ \\ ${ }^{a}$ Department of City and Regional Planning, Izmir Institute of Technology, 35430 Urla, Izmir, Turkey \\ ${ }^{\mathrm{b}}$ Artificial Iytelligence \& Design Lab., Department of Mechanical Engineering, Izmir Institute of Technology, 35430 Urla, Izmir, Turkey
}

\section{H I G H L I G H T S}

- Platoon-based formations are discussed through nonextensive thermostatistics.

- The limit values of Tsallis $q$ index for vehicular platoon formation are proposed.

- Superstatistics approach is utilized to obtain Tsallis $q$ index.

\section{A R T I C L E I N F O}

\section{Article history:}

Received 22 August 2016

Received in revised form 9 November 2016

Available online 22 December 2016

\section{Keywords:}

Nonadditivity

Tsallis entropy

Platoon formation

Superstatistics

Traffic flow

\begin{abstract}
A B S T R A C T
Many systems in nature and engineering are composed of subsystems. These subsystems may be formed in a linear, planar or spatial array. A typical example of these formations is a chain of vehicles known as platoon formation in traffic flow. Platoon formation of vehicles is a linear or planar formation of vehicles where a certain and a constant headway, and sideway if applicable, is provided in between every and each one of them. It is argued in this paper that a well-automated platoon formation of vehicles is an extreme case of conformity. During this transformation from a many degrees of freedom formation to a solid object, Tsallis $q$ value is computed to be ranging from one extreme case of $q=3$ to the other where $q=1$, when classified in terms of inverse temperatures of clearance fluctuations. At one extreme of $q=3$, one observes unbounded fluctuations in clearance fluctuations so that inverse temperature distributions approach a Dirac delta at the origin. At the other extreme of $q=1$, fluctuations in clearance tend to zero asymptotically, where a solid structure of agents (vehicles) emerges. The transition from $q=3$ to $q=1$ is investigated through synthetic and experimental clearance fluctuations between the cars. The results show that during the transition from $q=3$ to $q=1$, the platoon loses its many degrees of freedom (dof) of motion until a solid single object emerges. Authors assert that the Tsallis $q$ value of a platoon of vehicles is limited to $3>q>1$.
\end{abstract}

(c) 2016 Elsevier B.V. All rights reserved.

\section{Introduction}

Traffic flow is a complex, many particle system where vehicles come into contact, forming possibly long-range, nonMarkovian type memory, where the dominating form of thermostatistics is the nonextensive entropic domain, instead of the highly idealized Boltzmann-Gibbs (BG) framework. The existence of such interactions has been shown in detailed analysis

\footnotetext{
* Corresponding author.

E-mail addresses: cglrksn@gmail.com, caglarkosun@iyte.edu.tr (C. Kosun), serhanozdemir@iyte.edu.tr (S. Ozdemir).
} 
in [1,2]. Unless interactions are ignored, and simplified, traffic flow would hardly harbor Gaussian distributions, and ergodic time series.

Since resources in traffic are quite limited, the general tendency is to have a broader cooperation among the vehicles so that large groups of vehicles are commonly controlled and moved, avoiding jams, hoping to improve the overall efficiency of the traffic infrastructure. This coordinated and harmonious motion of vehicles is meant to have a better control of the highway traffic, directed to such amenities as fluent traffic, fuel economy, automated control of vehicles, etc. One such push is by a platoon formation where the vehicles are formed in a planar array, as well as a linear fashion. A prescribed distance is provided for each inter-vehicle clearance, and this distance and the speed of the formation are intended to be kept constant throughout and their corresponding fluctuations (errors) should be kept as small as possible. One may discuss the errors in either the speed, headway, sideway, or some of these variables for a given platoon formation. As the system is better controlled, the headway fluctuations, for example, is expected to subside, giving way to an almost constant specified headway distance. As the mean of the inverse variance and the variance of the inverse variance obtained from the errors in clearances tend to zero, it is seen that, with an idealized many body system, pdf of the inverse variance approaches the Dirac delta function.

The current traffic platoon formation literature has brought about multifarious topics, covering a wide range of issues. The major drive behind the platoon formation research has been an efficient and well-orchestrated traffic flow. Typical examples of the literature on the vehicular traffic platoon have concentrated on such topics as the stability [3-6], management and control [7,8], signal timing [9-11], safety, fuel consumption [12,13].

Of these traffic platoon literature for example the study [8] reviews some of the existing approaches to vehicular formation control under leader-follower approach and virtual leader approach, and in the paper the authors point to the importance of formation control in cooperative systems. The objective in their study is to design a feedback-based protocol for connected autonomous vehicles considering the different network topologies of initial states. Lyapunov technique is utilized to analyze the stability and consensus of the control protocol.

Another representative recent study for platoon management protocol could be [7] where the management is based on wireless communication. The protocol is designed under three maneuvers i.e. merge, split, and lane-change. The fuel consumption issue is addressed in many studies such as [13]. The authors consider a platooning problem in connection with minimizing the fuel consumption of the trucks, and the optimal platoon routing is studied in the paper. The study [14] deals with the multi- platoons-based cooperative driving and the inter-vehicle communication is the main concern in the study. The consensus based control algorithms are proposed for the vehicle platooning formulation. More than one platoon are considered and the results on inter and intra-platoon performances e.g. position and speed errors are evaluated for the system performance in the paper.

In another recent work [15] for the string stability problem of vehicle platoon, a collision prevention pre-compensation algorithm is proposed. The effectiveness of algorithm is tested by several simulation experiments. In the algorithm, such inputs as acceleration, deceleration, speed, time delay of vehicle-to-vehicle communication are introduced.

In the study of [16], the authors concentrated on two models of platoon formation over traffic flow breakdown at an expressway bottleneck, and speed transitions for a given platoon. Their traffic flow model considers the stochastic and dynamic processes of the traffic at the bottleneck. In their models probabilistic nature of traffic is investigated and, for example, for the speed transitions, Markov chain model is applied and on a bottleneck section of a single lane expressway, their combined platoon-based traffic flow model estimates the breakdown probability in regard to given flow rate.

The study of [5] deals with intra-platoon information management strategies, and in the study the platoon string stability is handled under the effects of communication delays. For the string stability in the platoon inter-vehicle communication is needed where the wireless communication is utilized. Five information updating schemes are proposed in which the leader vehicle conveys the data to its precedents and finally the simulation scenarios were also implemented.

In another work [6], the authors propose leader-following consensus algorithm to satisfy the string stability over the platoon of the vehicles. Spacing error is the main concern in the algorithm and it is computed between each vehicle and its neighbors. The simulation results cover the relation between both link probability and platoon size with error propagation, and the different communication graphs are also given for their algorithm.

The Ref. [17] studies $N$ identical particle (vehicles) on a circle, and deals with thermal equilibrium spacing distributions. Hamiltonian function is performed to describe the energy of the system. Spacing between neighboring vehicles, and velocity values of the vehicles are processed via a Hamiltonian function. However, the interactions are considered to be short-range, and the generalized thermostatistics approach is not discussed as in [18].

As one may easily notice, the related platoon literature governs for example, the inter-vehicle communication, stability, fuel consumption, control, etc. in traffic flow. In this paper, the cooperation in platoon formation is discussed through a broader framework i.e. nonextensive thermostatistics. No references in literature could be found addressing the platoon formation problem via nonextensivity, and to the best knowledge of the authors, this is the first time nonextensive thermostatistics is applied to the platoon formation in the literature. We propose an upper and a lower limit of Tsallis $q$ entropic index for platoon formations of interacting and cooperating agents. The authors first investigate the vehicular traffic platoon formation through a hypothetical scenario, and clearance errors are examined for the transition in the $q$ values. Then, a platoon of three vehicles is devised, equipped with ultrasonic range sensors, data acquisition cards and on-board data loggers. The synthetic and experimental findings are compared and later, results are discussed. The authors elaborate this so-called upper and lower limit theoretically in Section 3, whereas in Section 4 the authors utilize the superstatistics 
approach to obtain the $q$ values, taking into account chi-square distributed inverse pseudo temperatures. It is argued that when a maximum cooperation is observed, intra-platoon entropy would attain a minimum paradoxically at $q=1$ in solid form, when the platoon thermostatistics is governed by BG one. However, once the clearance fluctuations increase, the entropic index of the platoon tends to $q=3$. The reader should note that the computed $q$ values are gathered from the inverse temperature distributions.

\section{Superstatistics}

Possible generalization of BG theory has been proposed by Tsallis in 1988 [19]. It is also known as the generalization of conventional statistical mechanics, and it is referred to as nonextensive statistical mechanics. It covers the extensive BG thermostatistics as well. Often real systems may not be simplified and formulated under the assumptions of BG thermostatistics. Since these systems are generally multicomponent and complex, and interactions are non-local and dependent, relevant probability distributions of them deviate from the Gaussianity. Tsallis entropic form Eq. (1) is provided as below

$$
S_{q}=k \frac{1-\sum_{i=1}^{W} p_{i}^{q}}{q-1} \quad\left(\sum_{i}^{W} p_{i}=1 ; k>0\right)
$$

where $k$ is the Boltzmann constant, $q$ is the entropic index, and $p_{i}$ is the state probability.

$q-1$ could be considered to be an indicator of the deviation from the Gaussianity.

The dynamics of nonequilibrium systems could be represented with superstatistics theory. A superstatistical system could arise from the superposition of at least two statistics which would correspond to Boltzmann factor Eq. (2), $e^{-\beta E}$ and fluctuating intensive parameter $\beta$. The intensive parameter could be inverse temperature or another possible quantity such as pressure and chemical potential for the given driven nonequilibrium systems [20]. The parameter $\beta$ varies window to window in a given time scale, and hence a relevant probability distribution could be found. In superstatistics, common probability distributions out of $\beta$ fluctuations are log-normal, inverse chi-square, and chi-square. Once the $\beta$ is distributed according to chi-square distribution, then the nonextensive Tsallis statistics emerges [21].

The function of an averaged Boltzmann factor $(B F)$ in [21]

$$
B F(E)=\int_{0}^{\infty} f(\beta) e^{-\beta E} d \beta
$$

where $E$ is the energy, $f(\beta)$ is the probability distribution of $\beta$.

In superstatistics, the marginal probability $p(v)$ of data $v$ is given as, $[2,21]$

$$
p(v)=\int f(\beta) p(\nu \mid \beta) d \beta .
$$

In Eq. (3), once the $f(\beta)$ is $\chi^{2}$ distributed and the $p(v \mid \beta)$ is a Gaussian, then the $p(v)$ becomes a Tsallis distribution.

Under $\chi^{2}$ distributed $\beta$, the generalized canonical distributions of nonextensive statistical mechanics become, Eq. (4), $[2,21]$

$$
p(v) \sim\left(1+\frac{1}{2} \beta^{\circ}(q-1) v^{2}\right)^{\frac{1}{1-q}}
$$

where $\beta^{\circ}$ represents inverse variance of the whole data.

In our study, the intensive parameter can be described as inverse pseudo temperature which is derived from variances in the form of $1 / \sigma^{2}$.

\section{Methodology}

It is intended to formulate the limiting case or cases for linear or planar platoon formations of vehicles. For the perfectly controlled vehicle formations, one expects the variance of clearance error distribution of vehicles to tend to zero, as this will be used for the superstatistics approach.

Definition 3.1. Let the platoon formation is characterized by the states clearances $(C)$, sideway $(S)$, and the speed $(V)$, so that $Q(C, S, V) \in R^{(m-1) \times(n-1) \times k}$ is the combined state vector, Fig. 1, where an $m$-by- $n$ platoon as a many-body system has $(m-1) \times(n-1)$ planar degrees of freedom and $k$ represents the total number of vehicles. Then the general planar platoon formation, hence, $P(C, S) \in R^{(m-1) \times(n-1)}$ may be described as a matrix. 


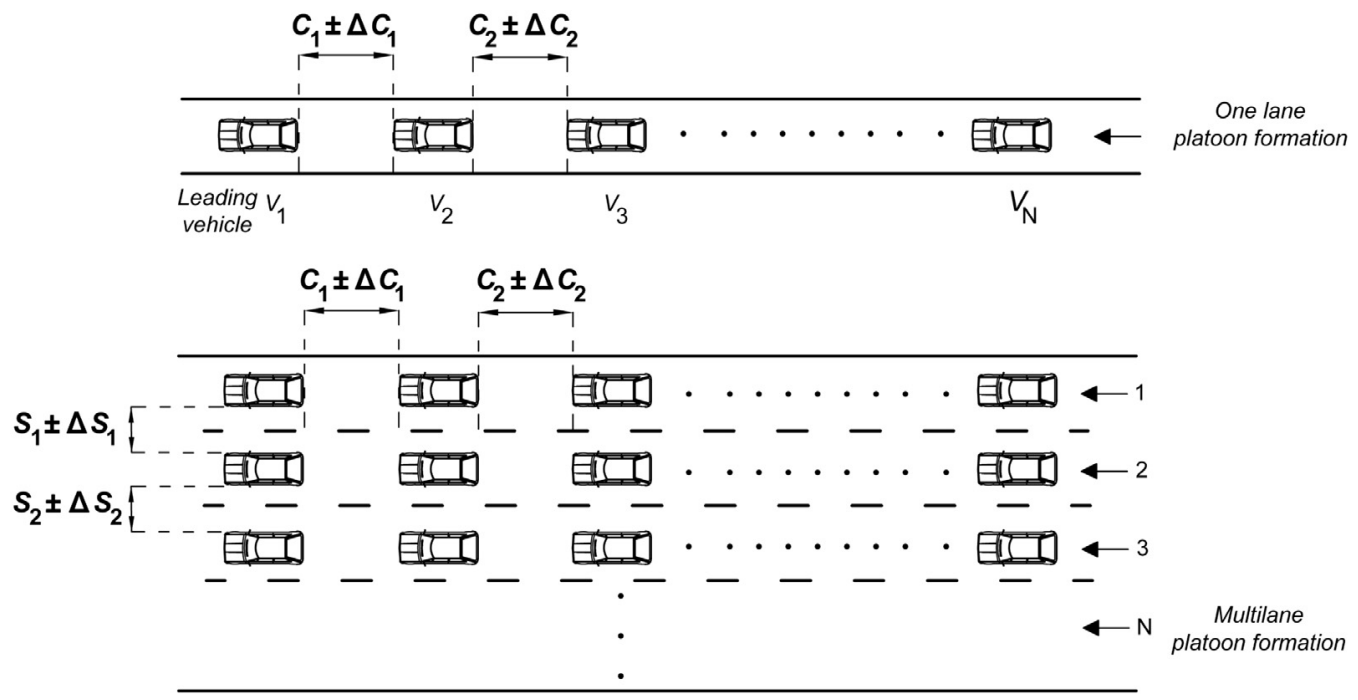

Fig. 1. Representation of one lane and multilane vehicular platoon formation.

Definition 3.2. Let $\{X\} \rightarrow R$ be a clearance error dataset of the uniformly distributed vehicles in a platoon formation, with variance $\operatorname{Var}(X)$ and mean $\mu(X)$. The clearances data is the set of the specified clearance and their respective errors.

Lemma 3.1. As the clearance errors tend to infinity, all of those unimodal continuous inverse temperature distributions approach a single distribution, $\delta(x)$, the Dirac delta distribution, whose width is shrunk to zero.

Lemma 3.2. For a stable platoon formation, the Dirac delta function is localized at the specified clearance $C$, with a fluctuation approaching zero. As $\mu(X) \rightarrow 0$ and $\operatorname{Var}(X) \rightarrow 0$, the resulting inverse temperature distribution may safely be regarded as a chisquare distribution $\chi^{2}$ with $d f \rightarrow \infty$, where, $d f$ is degrees of freedom of the chi-square distribution, by virtue of the Lemma 3.1.

Definition 3.3. Let the ideal platoon formation be approached in the limit. Then the general planar platoon formation, $P(C, S) \in R^{(m-1) \times(n-1)}$ where an $m$-by- $n$ platoon body would act as a single body, resulting in zero degrees of freedom of motion solid object in plane.

Theorem 3.1. As ideal platoon formation is approached, errors in clearances (also in sideways) tend to zero. Then successive clearance error readings are expected to locally relax to equilibrium at longer intervals. This state is the limiting case for any multi-component system, where all the degrees of freedom of motion is lost at $q=1$, obtained from the inverse temperature distribution. For all the real platoon formations, $q>1$.

Theorem 3.2. As collision avoidance is observed, and the maximum allowable fluctuations are assumed by the vehicles, the maximum variance in clearances appears. The distribution of inverse temperatures is trivial, $\delta(x)$. This state is the limiting case for any multi-component system, where this is the maximal degrees of freedom of motion with $\operatorname{Var}(X) \rightarrow \infty$, since all the vehicles are actively error generating. But speaking of a fit in superstatistics domain, it is an extreme case of zero degrees of freedom fit to a chi-square distribution, where $\mu(\beta) \rightarrow 0$. It is the opposite case of Theorem 3.1. This state of the platoon generates maximal error fluctuations where the $q$ value obtained from the fit to the inverse temperatures yields 3.

Proof. For a poorly controlled many-component system i.e. large fluctuations in clearance, headway, sideway, or speed data would generate $\operatorname{Var}(X)=\infty$ in the limit, and the superstatistical processing of the inverse temperatures would yield both $\operatorname{Var}(\beta)=0$ and $\mu(\beta)=0$. By virtue of Lemma 3.1, the distribution of the inverse temperature is a chi-square distribution with zero degrees of freedom. By $[21,22]$

$$
q \triangleq 1+\frac{2}{1+d f}
$$

where, $d f$ is degrees of freedom and zero in this case.

Eq. (5) dictates that the Tsallis index $q$ of maximal error variance platoon formations be at worst $q=3$ out of inverse temperature fit, but all the real systems be forced to $3>q$.

The authors hence tentatively propose a Tsallis $q$ index interval $3>q>1$ for platoon-based vehicle or any other agent formations, the Kosun-Ozdemir platoon interval. 
Lemma 3.3. The inequality $3>q>1$ represents only the intra-platoon interactions. Extra-platoon interactions must be regarded separately, either between the platoon and the outside world, or individual agents and the outside world.

Lemma 3.4. The variance in errors in clearances, sideway or speed data in the relevant phase space may only be reduced to zero in the limit by notifying all the agents in the formation simultaneously, should there be a traffic contingency that may affect the current state of the agents. All the communications and control actions must be immediate and platoon-wise global. Time constants, rise and settling times, if any, are all assumed to be zero.

Lemma 3.5. At $q=1$, this state is an extremum for the entropy, corresponding to a minimum. For the distinction of states, position or energy-wise, see [1]. At this limit, the platoon does not generate entropy, except for the leading vehicle. The number of the possible complexions for the rest of the vehicles is simply one. The leading vehicle represents the platoon and the platoon as a whole may act as a random object.

Lemma 3.6. Provided that the highway is occupied only by the platoon, and no other traffic around, in the limit, only the leading agent generates an entropy, and that entropy may simply be describable by BG thermostatistics, and nonextensive one may safely be ignored.

It should be stated that all the presented $q$ values are extracted through inverse temperature distribution generated by the theory of superstatistics. The limiting cases represented by the $q$ values in the preceding results may be seen contradictory to the reader. For example, as asserted in Theorem 3.1, an ideal highly coordinated platoon formation is characterized by $q=1$. But traditionally, the realm of $q=1$ is the domain of BG thermostatistics where the properties such as cooperation, loss of degrees of freedom of motion etc. are absent.

The authors argue that the appearance of the limiting case of $q=1$ corresponds to a single object platoon formation since this state of formation is also the limiting case where all the intra-platoon interactions cease to exist. As the whole platoon now becomes a single object, this single object may pick up a state randomly in its relevant phase space. The limiting case of $q=3$ corresponds to traffic flow involving high clearance fluctuations, whereas one might have expected instead the statistics of these fluctuations comply with BG formalism. In contrast to the single solid object case, now the particles are seemingly independent from each other. Please note again that these apparently contradicting $q$ values stem from the inverse temperature distribution.

\section{Experimental setup, analyses and results}

In the investigation of the transition from $q=3$ to $q=1$, synthetic and experimental data are utilized and their results are compared. In Section 3, it is claimed and theoretically shown that if the platoon formation had lost its many degrees of freedom, a Tsallis $q$ value of 1 would have been obtained. First, three chi-square distributions of synthetic clearance errors were generated. In all the cases, the maximum clearance error was reduced gradually from roughly a maximum of $100 \mathrm{~cm}$, to $60 \mathrm{~cm}$ and finally to $15 \mathrm{~cm}$. Inverse pseudo temperature distributions are provided as bars, Fig. 2. In experimental work, error ranges were observed by monitoring real-time clearance data inside the second and the third vehicles. Thus, both the theoretical and experimental analyses are conducted within the confined ranges of $\pm 100, \pm 60$, and $\pm 15 \mathrm{~cm}$ clearance error data.

For the theoretical analysis, a maximum of $\pm 100 \mathrm{~cm}$ error on synthetic clearance data has been generated and their inverse temperature values $\beta$ are fitted with roughly 0.01 degrees of freedom, then the $\beta$ values from $\pm 60 \mathrm{~cm}$ error has produced roughly 0.02 degrees of freedom fit, and finally $\beta$ values from $\pm 15 \mathrm{~cm}$ error yielded 2.2 , Fig. 2. Approximately, no smaller than $\pm 15 \mathrm{~cm}$ data could have been processed, since data distribution has become quite biased, and the analysis of superstatistics could no longer be reliable based on $\varepsilon, \tau / T$ superstatistical criteria [2,23]. Besides, Fig. 5 is simply a representation of the current trend (Lemma 3.1). Since the $\beta$ parameter was scaled, it is easy to track the evolution of the size and location of the bars as $d f$ comes down. It is seen that it is compressed towards left, Figs. 2 and 4.

As it can be inferred from Fig. 2, for the $\pm 100 \mathrm{~cm}$ error range, $\beta$ parameter values fall into the $0.004-0.014$ range, then the histogram peak occurs markedly since much of $\beta$ values shrink into very small interval. For this range, Dirac deltalike probability distribution is in question where the $0.01 d f$ chi-square distribution could be well fitted. According to the analyses of $\pm 60 \mathrm{~cm}$ errors, the $\beta$ values on the histogram are in the range of $0.008-0.04$, the form of Dirac delta probability distribution begins to dissolve. The smallest range corresponds to $\pm 15 \mathrm{~cm}$ error, and the $\beta$ values on the histogram are located in broader interval, which corresponds to 0.03-0.5.

Our findings in this paper indicate that, for the platoon formation once the clearance errors are generated with high degrees of freedom chi-square distribution, the resulting $\beta$ distributions, in turn, exhibit low degrees of freedom chisquare distribution. In contrast, the given distribution of clearance errors with low degrees of freedom would lead to the extraction of the $\beta$ distribution with high degrees of freedom. Furthermore, a lessening of degrees of freedom represents the diminishing of clearance errors, which also ensures the decreasing Tsallis entropic index $q$ obtained from inverse temperatures. Thus, the obtained $q$ values are roughly 1.62, 2.96, and 2.98 through the computed degrees of freedom from $\beta$-fit which are $2.2,0.02$, and 0.01 , respectively as provided by Eq. (5). When inquiring the influence of range of clearance errors on Tsallis $q$ values, it is interpreted that the change in \pm 60 to \pm 15 is highly effective on the decrease in $q$ value. In a 
Table 1

The parameters of three different synthetic clearance error data.

\begin{tabular}{lllll}
\hline Synthetic data & Error range $(\mathrm{cm})$ & df of $\beta$-fit & Computed $q$ value & Inverse temperature range \\
\hline Clearance error data \# 1 & \pm 100 & 0.01 & 2.98 & $0.004-0.014$ \\
Clearance error data \# 2 & \pm 60 & 0.02 & 2.96 & $0.008-0.04$ \\
Clearance error data \# 3 & \pm 15 & 2.2 & 1.62 & $0.03-0.5$ \\
\hline
\end{tabular}

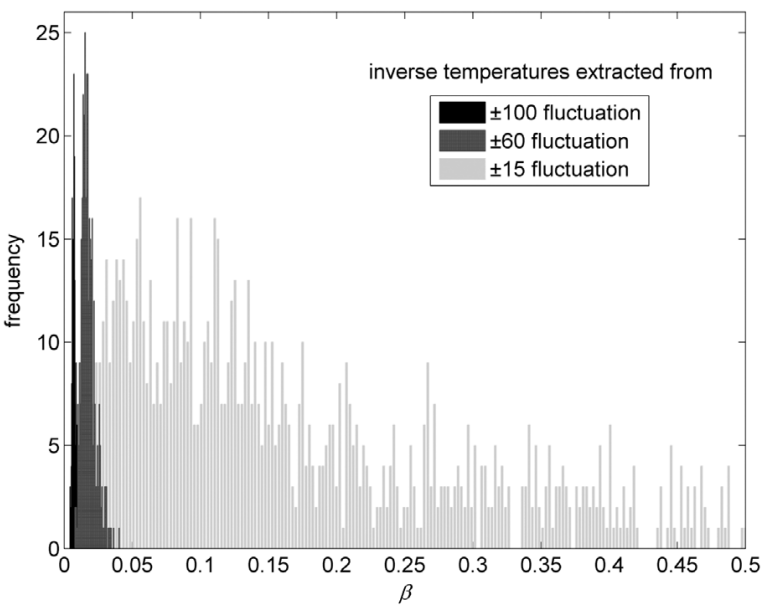

Fig. 2. Histogram of inverse temperatures from synthetic clearance error data.

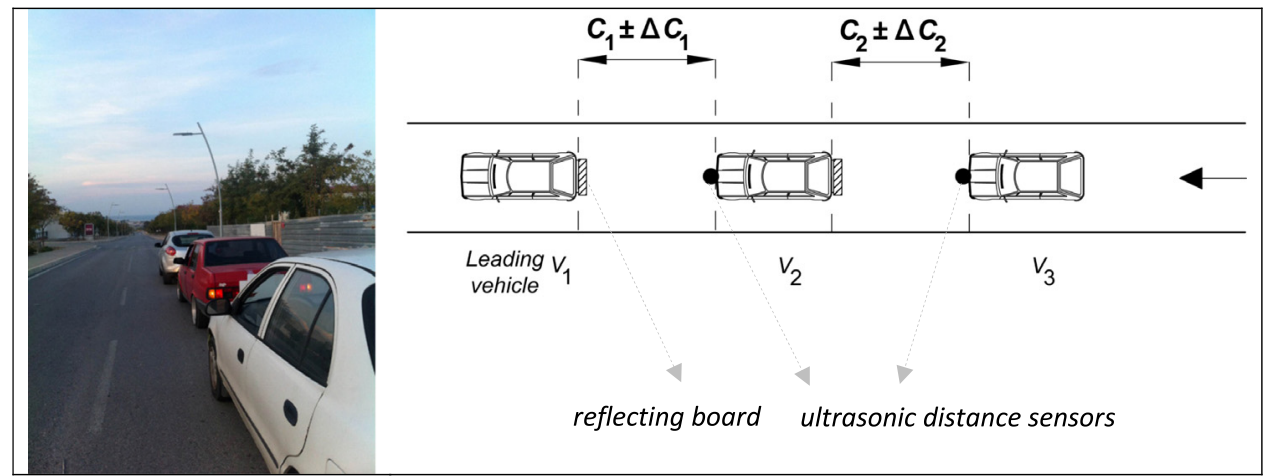

Fig. 3. The photograph and the illustration of actual in-campus platoon experiment.

nutshell, the change in computed $q$ values is more pronounced between data \# 2 and data \# 3 than between data \# 1 and data \# 2 . The results are summarized on Table 1.

A series of actual platoon tests were conducted to judge how well the theoretical conclusions match those of real traffic platoon (see Fig. 3). Four separate drives with three different cars in a single lane were carried out. Each drive is approximately $1 \mathrm{~km}$ long, and vehicle speeds were limited to approximately $20 \mathrm{~km} / \mathrm{h}$ to prevent possible collisions. The experiment was conducted in the university campus, and during the tests driving vehicles were forced to occasional speed changes due to pedestrian crossings and speed bumps, which are quite visible in histograms as noise. Three cars are involved in the experiments, and second and third vehicles were equipped with ultrasonic distance measuring sensors. The clearance readings are recorded. The superstatistical analyses are carried out, and the inverse temperature values are calculated for each clearance error interval and plotted in Fig. 4. Empirical results assert that $\pm 100 \mathrm{~cm}$ clearance error range generates the inverse temperature probability distribution à la Dirac delta on the left of Fig. 4, approaching $q=3$. On the other hand, those of $\pm 15 \mathrm{~cm}$ clearance error range provide growing variance with a decrease in $q$.

Our analyses indicate that the inverse temperature values for the \pm 100 and \pm 60 clearance errors from the synthetic data shrink into the lower intervals compared to the actual data, while those for the \pm 15 clearance errors become wider. This also proves that synthetic data would make the $q$ values of inverse temperatures stretch to the limits. The degrees of freedom values rise from 0.35 to 0.6 and then finally to 1.2 ., for the $\pm 100, \pm 60, \pm 15$ experimental clearance error data, respectively, Table 2. Hence, the computed $q$ values for the actual tests are between 2.48 and 1.9, Eq. (5), whereas those out of synthetic data varied between 2.98 and 1.62, showing broader interval of the $q$ values for the synthetic one. 
Table 2

The parameters of three different experimental clearance error data.

\begin{tabular}{lllll}
\hline Experimental data & Error range $(\mathrm{cm})$ & df of $\beta$-fit & Computed $q$ value & Inverse temperature range \\
\hline Clearance error data \# 1 & \pm 100 & 0.35 & 2.48 & $0.0026-0.08$ \\
Clearance error data \# 2 & \pm 60 & 0.6 & 2.25 & $0.0043-0.1484$ \\
Clearance error data \# 3 & \pm 15 & 1.2 & 1.9 & $0.0446-0.1830$ \\
\hline
\end{tabular}

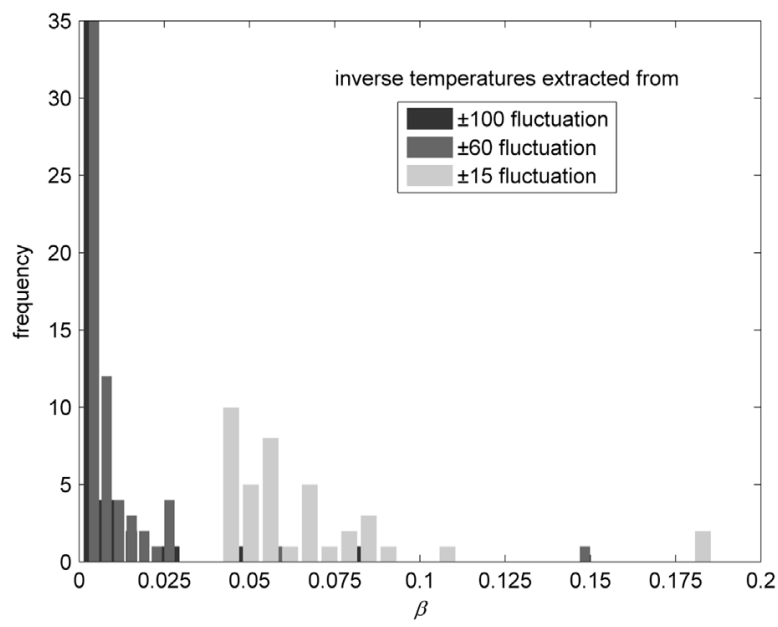

Fig. 4. Histogram of inverse temperatures from experimental clearance error data.

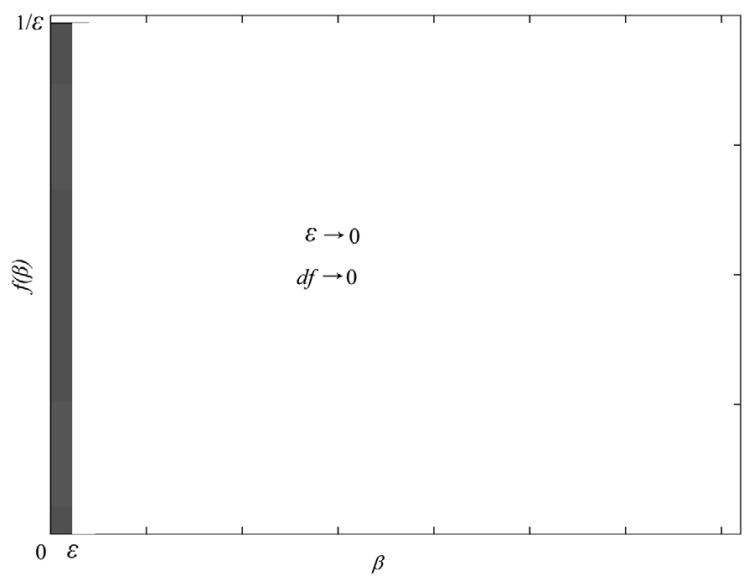

Fig. 5. Histogram of inverse temperatures of clearance errors.

It should be stressed out that the assertion that as the fluctuations tend to infinity inverse temperature distributions aggregate on the left around zero towards a Dirac delta distribution has been verified, both theoretically, Fig. 2, and in practice Fig. 4. It is also shown that as fluctuations tend to zero, inverse temperature distributions migrate to the right. Inspecting Figs. 2 and 4 reveals that theoretical data is spread out further than the actual data obtained from a series of experiments of a platoon of three vehicles, Fig. 4. The spreading of the inverse temperatures out of $\pm 15 \mathrm{~cm}$ error fluctuations is more apparent in both synthetic and actual data. This would translate into smaller $q$ values compared to those of larger fluctuation data, as evident on Tables 1 and 2.

\section{Conclusions}

The limiting cases of linear or planar formations of vehicular platoons have been discussed in the context of nonextensive thermostatistical framework. An idealized scenario is considered where a perfect control of a platoon of vehicles is devised. In this scheme, the inter-vehicle clearances, and possibly sideways for that matter, are kept constant. This hypothetical scenario also allowed zero or near zero errors in clearances, tending to infinite degrees of freedom of fit to inverse temperature distributions in the relevant superstatistical analyses. 
The platoon problem is presented through a set of lemmas and theorems, to propose the limiting cases of this perfectly controlled, highly coordinated, near zero entropy vehicular cluster, and its opposite high variance, loose set of vehicles, reminiscent of gas state. It is also conjectured that well-coordinated and controlled many body systems lose degrees of freedom, until finally a rigid body state is reached where $q=1$.

By superstatistics, it is proven that a zero degree of freedom chi-square distribution must approach the limiting case of $q=3$, where the platoon has the maximal degrees of freedom. Through superstatistics, it is shown that, for unimodal distributions, since the Dirac delta function is a limiting case of distributions, it must possess a zero degree of freedom, having a zero mean and the variance.

The proposed limiting cases of $q$ may be seen conflicting. Even though the realm of $q=1$ is the domain of BG thermostatistics, an ideal highly coordinated platoon formation has been characterized by $q=1$. At the other extreme, the limiting case of $q=3$ corresponds to platoon traffic flow involving high clearance fluctuations where one might have expected an ordered traffic flow.

The authors would like to generalize the lower limit of the proposed interval platoon-like traffic flow to any wellorganized many-body system where similar interacting and cooperating agents may be observed, such as swarms of robots on a specified mission. Since the limiting cases are also an indicator of the level of cooperation, it may very well be emulated for resource management.

\section{References}

[1] C. Kosun, The Use of Nonextensive Framework in connection with Traffic Flow [Ph.D. dissertation], Department of City and Regional Planning, Izmir Institute of Technology Graduate School of Engineering and Sciences, Izmir, 2015.

[2] C. Kosun, S. Ozdemir, A superstatistical model of vehicular traffic flow, Physica A 444 (2016) 466-475

[3] Y. Li, H. Zhu, M. Cen, Y. Li, R. Li, D. Sun, On the stability analysis of microscopic traffic car-following model: a case study, Nonlinear Dynam. 74 (1-2) (2013) 335-343.

[4] J. Ploeg, N. Van De Wouw, H. Nijmeijer, Lp string stability of cascaded systems: Application to vehicle platooning, IEEE Trans. Control Syst. Technol. 22 (2) (2014) 786-793.

[5] P. Fernandes, U. Nunes, Platooning with IVC-enabled autonomous vehicles: Strategies to mitigate communication delays, improve safety and traffic flow, IEEE Trans. Intell. Transp. Syst. 13 (1) (2012) 91-106.

[6] Y. Ruan, S.K. Jayaweera, Leader-following consensus in vehicle platoons with an inter-vehicle communication network, in: The 8th International Conference: Telecommunication Systems Services and Applications, TSSA, Kuta, 2014

[7] M. Amoozadeh, H. Deng, C.N. Chuah, H.M. Zhang, D. Ghosal, Platoon management with cooperative adaptive cruise control enabled by VANET, Veh. Commun. 2 (2) (2015) 110-123.

[8] Y. Li, K. Li, T. Zheng, X. Hu, H. Feng, Y. Li, Evaluating the performance of vehicular platoon control under different network topologies of initial states, Physica A 450 (2016) 359-368.

[9] Q. He, K.L. Head, J. Ding, PAMSCOD: Platoon-based arterial multi-modal signal control with online data, Transp. Res. C 20 (1) (2012) 164-184.

[10] X.F. Xie, G.J. Barlow, S.F. Smith, Z.B. Rubinstein, Platoon-based self-scheduling for real-time traffic signal control, in: The 14th International IEEE Conference: Intelligent Transportation Systems, ITSC, Washington DC, 2011.

[11] Y. Jiang, S. Li, D.E. Shamo, A platoon-based traffic signal timing algorithm for major-minor intersection types, Transp. Res. B 40 (7) (2006) 543-562.

[12] J. Larson, C. Kammer, K.Y. Liang, K.H. Johansson, Coordinated route optimization for heavy-duty vehicle platoons, in: The 16th International IEEE Conference: Intelligent Transportation Systems, ITSC, The Hague, 2013.

[13] E. Larsson, G. Sennton, J. Larson, The vehicle platooning problem: Computational complexity and heuristics, Transp. Res. C 60 (2015) $258-277$.

[14] D. Jia, D. Ngoduy, Platoon based cooperative driving model with consideration of realistic inter-vehicle communication, Transp. Res. C 68 (2016) $245-264$.

[15] J. Zhang, X. Jia, Z. Zhou, A pre-compensation control algorithm for vehicle platoon stability problem, Optik-Int. J. Light Electron Opt. 126 (19) (2015) 2208-2213.

[16] Y. Shiomi, T. Yoshii, R. Kitamura, Platoon-based traffic flow model for estimating breakdown probability at single-lane expressway bottlenecks, Transp. Res. B 45 (9) (2011) 1314-1330

[17] M. Krbálek, Equilibrium distributions in a thermodynamical traffic gas, J. Phys. A 40 (2007) 5813-5821.

[18] M. Krbálek, D. Helbing, Determination of interaction potentials in freeway traffic from steady-state statistics, Physica A 333 (2004) $370-378$.

[19] C. Tsallis, Possible generalization of Boltzmann-Gibbs statistics, J. Stat. Phys. 52 (1-2)(1988) 479-487.

[20] C. Beck, E.G.D. Cohen, Superstatistics, Physica A 322 (2003) 267-275.

[21] C. Beck, Superstatistics: theory and applications, Contin. Mech. Thermodyn. 16 (3) (2004) 293-304.

[22] C. Beck, Dynamical foundations of nonextensive statistical mechanics, Phys. Rev. Lett. 87 (18) (2001)

[23] E. Van der Straeten, C. Beck, Superstatistical fluctuations in time series: Applications to share-price dynamics and turbulence, Phys. Rev. E (3) (2009). 